Edgardo Abelardo, MD,

Tetsuji Sanuki, MD, PhD,

Eiji Yumoto, MD, PhD

Department of Otorhinolaryngology

Head and Neck Surgery

Kumamoto University Hospital

Japan

\section{Medialization and Stabilization of the Middle Turbinate using a Nasal Septal Flap in Endoscopic Sinus Surgery}

\section{ABSTRACT}

Objectives: To propose a simple technique to preserve, medialize and stabilize the middle turbinate through a planned medial synechiae formation using a nasal septal flap

\section{Methods:}

a. Study Design: Case series, surgical innovation

b. Setting: Tertiary hospital (Kumamoto University Hospital, Japan)

c. Participants, Patients or Population: Six patients suffering from chronic sinusitis unresponsive to medication. The indication for doing this technique was unstable turbinates after removal of diseased mucosa.

Results: Twelve weeks after surgery, the patients reported significant resolution of symptoms (headache, nasal congestion, rhinorrhea, post-nasal drip and loss of smell). CT and endoscopic findings likewise revealed clear ostio-meatal complex, drained sinuses, and complete removal of polypoid mucosa. No major side effects were noted.

Conclusions: This technique is especially encouraged when surgeons encounter an unstable middle turbinate after removing massive pathologic mucosal lesions. This is a preliminary report and further investigations are being carried out to validate the technique.

\section{Keywords: medial synechiae, nasal septal flap, ostio-meatal complex}

LATERAL synechiae formation of the middle turbinate remains the most common complication in endoscopic sinus surgery. It results in recurrence of sinus symptoms due to blockage of the ostio-meatal complex and patient dissatisfaction. Some surgeons opt to partially resect the middle turbinate to ensure post-operative patency of sinus drainage but we consider this technique quite radical and contradictory to the conservative principle of functional endoscopic sinus surgery. In untrained hands, a planned partial resection may lead to total resection and loss of anatomic landmarks essential in revision surgery. Excessive middle turbinate manipulation may compromise such nasal functions as filtration, humidification and laminar flow. We propose a simple technique to preserve, medialize and stabilize the middle turbinate through a planned medial synechiae formation using a nasal septal flap.

\section{MATERIALS AND METHODS}

Records of patients in a tertiary hospital in Japan (Kumamoto University Hospital) who had endoscopic sinus surgery with middle turbinate medialization using septal flap from October 2001 to May 2003 were reviewed. The indication for the septal flap technique was unstable floppy middle turbinate after removing pathologic mucosal lesions. All cases had exposed middle turbinate bone on the lateral side with a high chance of developing lateral synechiae post-operatively. Only cases done by one surgeon (EY) were included in the study to avoid interoperator variability. Pre- and post-operative symptoms (headache, nasal congestion, rhinorrhea, post-nasal drip and loss of smell) were compared on a numerical scale which patients routinely accomplished during out-patient visits prior to, and at weeks 3 and 12 after surgery. Endoscopic findings and CT scan plates before, and 12 weeks after surgery were reviewed, as were intraoperative video recordings and major and minor post-operative complications. 


\section{SURGICAL TECHNIQUE}

The main indication for the medialization and stabilization technique using nasal septal flap was unstable and floppy middle turbinates after removal of diseased nasal mucosa. Following uncinectomy and widening of the maxillary ostium, the middle turbinate was medialized using a Freer elevator. An anteriorly-based rectangular nasal septal flap measuring $5 \mathrm{~mm} \times 15 \mathrm{~mm}$ was drawn adjacent to the de-epithelialized margin of the middle turbinate. The lower border of the middle turbinate marked the inferior margin of the septal flap. The superior margin of the flap was $5 \mathrm{~mm}$ above the inferior margin. Superior and inferior incisions were made with a sickle knife, subperichondrial dissection performed using a small Freer elevator, and the posterior margin was cut with scissors (Fig.1). The middle turbinate was medialized and the nasal flap hinged on its lateral side with the anterior border of the flap adjacent to the anterior border of the middle turbinate. The middle turbinate and flap were stabilized with fibrin glue (Fig. 2) and gentle nasal packing placed lateral to the middle turbinate for 48 to 72 hours.

When a bilateral medialization technique was indicated, the flaps on both sides of the septum were designed in such a way that septal cartilage was not exposed on the same level on both sides.

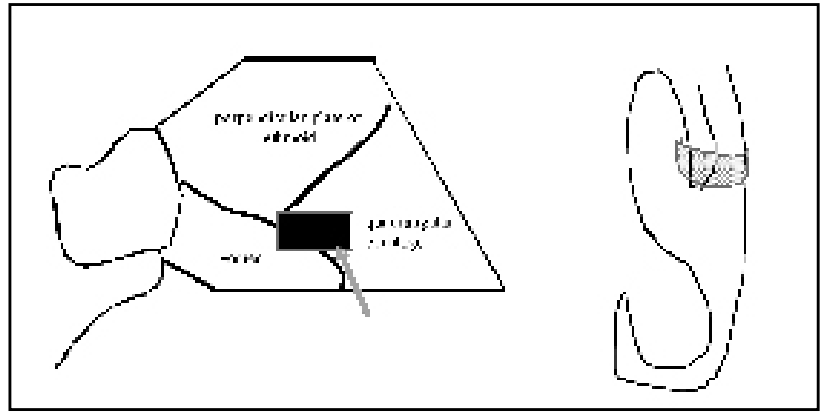

Figure 1. Schematic diagram. Lateral and anterior views of anteriorlybased nasal septal flap.

\section{RESULTS}

Six cases of middle turbinate medialization using septal flap were reviewed. There were 4 male and 2 female patients with an average age of 49 years. The main indication for surgery was chronic sinusitis. Two had pansinusitis, another two had maxillary sinusitis alone, and the remaining two had frontal sinusitis with mucoceole. Four had previous nasal surgeries, with one case previously done in another hospital whose post-operative records could not be retrieved.

Pre-operatively, all six patients (6/6) had symptoms of mild to severe post-nasal drip, five (5/6) reported mild to severe nasal congestion, mild to severe rhinorrhea, and mild to severe loss of smell, and four (4/6) had moderate headache. Endoscopy revealed pathologic nasal mucosa and obstructed ostio-meatal complex in five patients (5/6) and nasal polyps in three (3/6). These findings were confirmed by paranasal sinus CT scans. Table 1 summarizes the total symptom scores pre-op and postop (5-severe, 3-moderate, 1-mild, 0 -none; $n=6$ patients).

Twelve weeks after surgery, there were significant improvements in all of the symptoms (Figure 3). Five patients (5/6) reported improvement

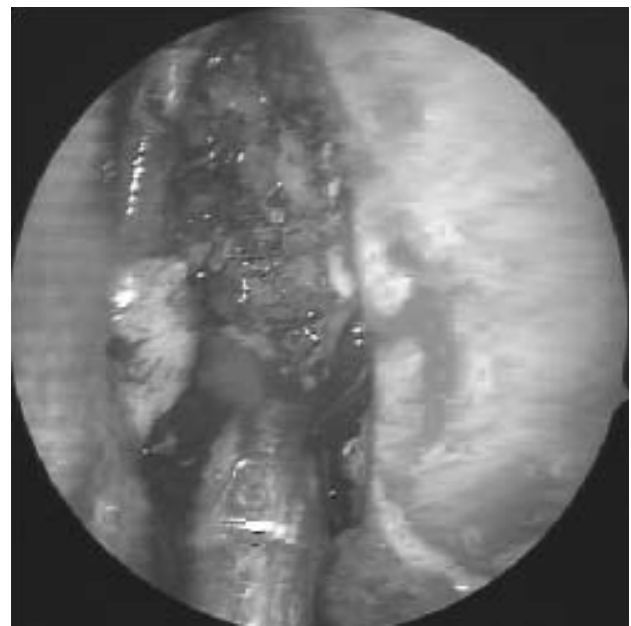

Figure 2. Intra-operative endoscopic view of left nostril after medialization of middle turbinate and stabilization using nasal septal flap.

Table 1. Total symptom scores

\begin{tabular}{l|c|c|}
\multicolumn{1}{|c|}{ Symptoms } & Pre-op & 12 weeks post-op \\
A. Headache & 12 & 3 \\
B. Nasal congestion & 15 & 3 \\
C. Rhinorrhea & 15 & 6 \\
D. Post-nasal drip & 18 & 3 \\
E. Loss of smell & 15 & 3 \\
$\quad$ CT/endoscopic findings & & \\
F. Obstructed OMC & 15 & 0 \\
G. Nasal polyp & 9 & 0 \\
\hline
\end{tabular}

(5-severe, 3-moderate, 1-mild, 0-none; $n=6$ patients); OMC-ostio-meatal complex).

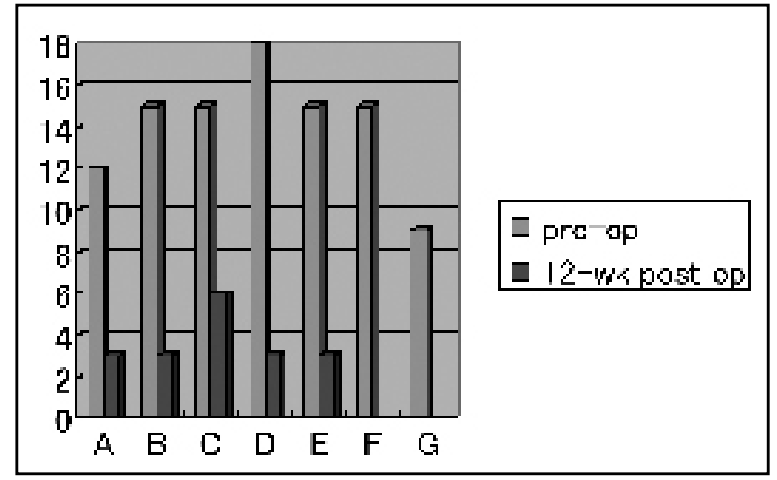

Figure 3. Bar graph of Table 1 showing pre-op and post-op symptoms and $\mathrm{CT} /$ endoscopic findings. (A-headache, B-nasal congestion, C-rhinorrhea, D-post-nasal drip, E-loss of smell, Fobstructed OMC, G-nasal polyp). 
in post-nasal drip, nasal congestion, headache and olfaction while one (1/6) claimed persistence of post-nasal drip, mild headache and mild nasal blockage. Severe to moderate rhinorrhea were downgraded to mild rhinorrhea in four patients (4/6). Endoscopic findings showed well-defined medial synechia and a wide ostio-meatal complex in all cases (Figs. 4, 5). Comparison of pre- and post operative CT scans showed widened ostio-meatal complexes in all cases and total removal of polypoid mucosa (6/6). No major complications were noted.

Statistical analysis could not be done at this stage because the sample size is small. The results should also be compared to the standard sinus surgery.

\section{DISCUSSION}

The middle turbinate plays an important role in endoscopic sinus surgery. Following extensive removal of pathologic tissue, it may be tempting to resect an unstable floppy middle turbinate to avoid lateralization and synechiae formation on the lateral wall with subsequent ostio-meatal complex obstruction and recurrence of nasal symptoms. Such resection of the middle turbinate removes a very important anatomic landmark in revision surgery.

Several techniques in medializing the middle turbinate have been proposed. Shikani advocated meatal antrostomy stents in the maxillary natural ostium for 2 weeks until re-epithelialization occured to keep the ostio-meatal complex patent ${ }^{1}$. Moukarzel used a metallic clip on the head of the middle turbinate and the septum ${ }^{2}$. Thorton suggested suturing of the middle turbinate and septum for stabilization ${ }^{3}$. Bolger described planned medial synechiae by abrading the middle turbinate and septum using a sickle knife and stabilizing the lateral side of the middle turbinate with packing ${ }^{4}$. Friedman furthered this idea, reporting excellent results using a microdebrider instead of a sickle knife ${ }^{5}$.

In our opinion, ostio-meatal complex stents and turbine-septal metallic clips may cause foreign body reactions and further granulation; turbino-septal suturing seems quite a difficult and time-consuming procedure; and sickle knife or microdebrider abrasion followed by nasal pack stabilization of the middle turbinate can cause imprecise apposition of the denuded surfaces. A tight nasal pack could displace the site of planned medial synechiae. Synechiae formation on the posterior segment of the middle turbinate could lateralize the anterior segment, causing ostio-meatal complex obstruction and recurrence of symptoms. Excessive nasal pack pressure could also medialize and cause synechiae of the entire medial segment of middle turbinate to the nasal septum, blocking airflow to the nasal roof leading to loss of smell. The nasal flap technique allows precise determination of the site of medial synechiae. An ideal medialization of the middle turbinate is optimally stabilized by planned small synechiae at its antero-inferior region leaving enough space at its antero-superior and posterior regions.

The technique we propose is an alternative to Friedman's procedure, cognizant of the fact that not all institutions have a microdebrider available. Moreover, this technique does not depend on nasal packing to medialize and stabilize the middle turbinate until a synechiae is formed. Instead, the planned site of medial synechiae is controlled
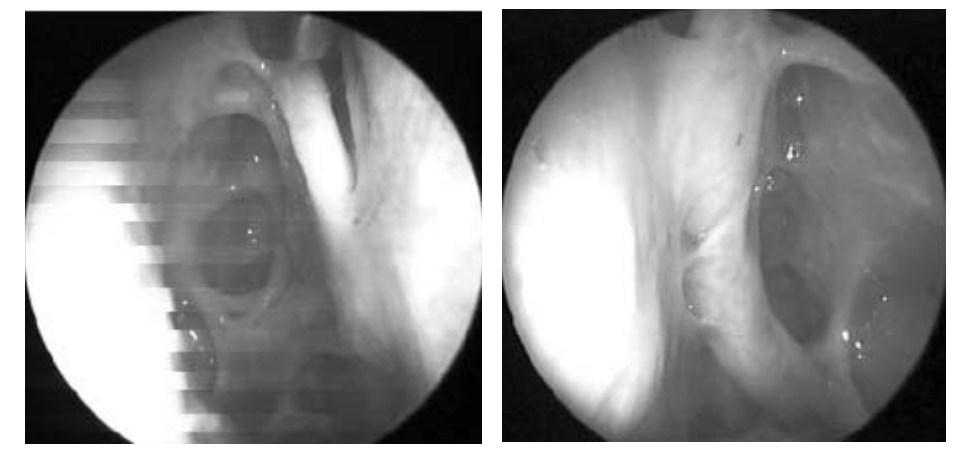

Figures 4 (above) and 5 (below). Right (4) and left (5) nostrils taken 3 months after surgery. Note the planned medial synechiae on the antero-inferior portion of the middle turbinate.

by the surgeon through the septal flap followed by gentle nasal pack application to avoid flap displacement. We think similar results could be achieved even without nasal packs.

Although middle turbinate medialization may theoretically result in hyposmia due to decreased airflow in the olfactory area, Friedman showed noadverse effect of medial synechiae on olfaction ${ }^{6}$. Furthermore, the improvement of sense of smell in most of our patients may be attributed to the wide space in the anterior and posterior segments of the middle turbinate allowing airflow to the spheno-ethmoid recess.

Our proposed technique is a quick and easy alternative to medialize and stabilize the middle turbinate without a microdebrider and using minimal nasal dressing. This technique is especially encouraged when surgeons encounter unstable middle turbinate after removing massive pathologic mucosal lesions. No major side effects were noted. This is a preliminary report and further investigation is being carried out to validate the technique and allow greater documentation of its effects on nasal function.

\section{REFERENCES:}

1. Shikani AH. A new middle meatal antrostomy stent for functional endoscopic sinus surgery. Laryngoscope 1994;638-41.

2. Moukarzel N, Nehme A, Mansour S, et al. Middle turbinate medialization technique in functional endoscopic sinus surgery. J Otolaryngol 2000 Jun;29(3):144-7.

3. Thorton RT. MT stabilization technique in endoscopic sinus surgery. Arch Otolaryngol Head Neck Surg 1996;122:869-72

4. Bolger WE, Kuhn FA, Kennedy DW. Middle turbinate stabilization after functional endoscopic sinus surgery. The controlled synechiae technique. Laryngoscope 1999;109:1852-3.

5. Friedman $M$, Lansberg $R$, Tanyeri $H$. Middle turbinate medialization and preservation in endoscopic sinus surgery. Otolaryngol Head Neck Surg 2000;123:76-80.

6. Friedman $M$, Tanyeri $H$, Lansberg $R$. Effects of middle turbinate medialization on olfaction. Laryngoscope 1999 Sep;109(9):1442-5. 